\title{
A review on nephrotic syndrome with their causes, complications and epidemiology
}

\author{
Monika Semwal* \\ Kukreja Institute of Pharmaceutical Sciences, Dehradun, U.K., India
}

*Correspondence

Monika Semwal

Kukreja Institute of Pharmaceutical Sciences, Dehradun, U.K., India.

Email: monikasemwal31@gmail.com

Received: 04-04-2020 / Revised: 02-05-2020 / Accepted: 15-05-2020

\begin{abstract}
Nephrotic syndrome is a collection of symptoms due to kidney damage. This includes protein in the urine, low blood albumin levels, high blood lipids, and significant swelling. Other symptoms may include weight gain, feeling tired, and foamy urine. Complications may include blood clots, infections, and high blood pressure. Causes include a number of kidney diseases such as focal segmental glomerulosclerosis, membranous nephropathy, and minimal change disease. It may also occur as a complication of diabetes or lupus. The underlying mechanism typically involves damage to the glomeruli of the kidney. Diagnosis is typically based on urine testing and sometimes a kidney biopsy. It differs from nephritic syndrome in that there are no red blood cells in the urine. Treatment is directed at the underlying cause. Other efforts include managing high blood pressure, high blood cholesterol, and infection risk. A low salt diet and limiting fluids is often recommended. About 5 per 100,000 people are affected per year. The usual underlying cause varies between children and adults.
\end{abstract}

Key words: Syndrome, Nephropathy, Biopsy and Glomerulosclerosis

This is an Open Access article that uses a fund-ing model which does not charge readers or their institutions for access and distributed under the terms of the Creative Commons Attribution License (http://creativecommons.org/licenses/by/4.0) and the Budapest Open Access Initiative (http://www.budapestopenaccessinitiative.org/read), which permit unrestricted use, distribution, and reproduction in any medium, provided the original work is properly credited.

\section{Introduction}

Nephrotic syndrome is a nonspecific kidney disorder characterized by three signs of disease: large proteinuria $\left(>3.5 \mathrm{~g}\right.$ per $1.73 \mathrm{~m}^{2}$ body surface area per day, or $>40 \mathrm{mg} \mathrm{m}^{2}$ body surface area per hour in children), hypoalbuminemia ( $<2.5 \mathrm{~g} / \mathrm{dl})$, and edema (which is generalized and also known as dropsy) that begins in the face [1]. Essentially, loss of protein through the kidneys (proteinuria) leads to low protein levels in the blood (hypoalbuminemia), which causes water to be drawn into soft tissues (edema). Lipiduria (lipids in urine) can also occur, but is not essential for the diagnosis of nephrotic syndrome. Hyponatremia also occurs with a low fractional sodium excretion. Very low hypoalbuminemia can also cause a variety of secondary problems, such as water in the abdominal cavity (ascites), around the heart or lung (pericardial effusion, pleural effusion), high cholesterol (hyperlipidemia), loss of molecules regulating coagulation (increased risk of thrombosis) [2].

Large proteinuria is due to an increase in permeability of the "filtering membrane" of the kidney which normally separates the blood from the urinary space in Bowman's capsule. This is composed of the capillary walls of the glomerulus which are wrapped by highly specialized cells called podocytes. Alterations in their capacity to filter the substances transported in the blood mean that proteins but not cells pass into the urine (no haematuria). By contrast, in nephritic syndrome red blood cells pass through the pores, causing haematuria [3].

\section{Causes}

Nephrotic syndrome has many causes and may either be the result of a glomerular disease that can be either limited to the kidney, called primary nephrotic syndrome (primary glomerulonephritis), or a condition 
that affects the kidney and other parts of the body, called secondary nephrotic syndrome [4].

Primary glomerulonephritis

Primary causes of nephrotic syndrome are usually described by their histology:

$>$ Minimal change disease (MCD): is the most common cause of nephrotic syndrome in children. It owes its name to the fact that the nephrons appear normal when viewed with an optical microscope as the lesions are only visible using an electron microscope. Another symptom is a pronounced proteinuria.

$>$ Focal segmental glomerulosclerosis (FSGS): is the most common cause of nephrotic syndrome in adults. It is characterized by the appearance of tissue scarring in the glomeruli. The term focal is used as some of the glomeruli have scars, while others appear intact; the term segmental refers to the fact that only part of the glomerulus suffers the damage.

$>$ Membranous glomerulonephritis (MGN): The inflammation of the glomerular membrane causes increased leaking in the kidney. It is not clear why this condition develops in most people, although an auto-immune mechanism is suspected.

$>$ Membranoproliferative glomerulonephritis (MPGN): is the inflammation of the glomeruli along with the deposit of antibodies in their membranes, which makes filtration difficult.

$>$ Rapidly progressive glomerulonephritis (RPGN): (Usually presents as a nephritic syndrome) A patient's glomeruli are present in a crescent moon shape. It is characterized clinically by a rapid decrease in the glomerular filtration rate (GFR) by at least $50 \%$ over a short period, usually from a few days to 3 months [5].

They are considered to be "diagnoses of exclusion", i.e. they are diagnosed only after secondary causes have been excluded.

\section{Secondary glomerulonephritis}

Secondary causes of nephrotic syndrome have the same histologic patterns as the primary causes, though they may exhibit some difference suggesting a secondary cause, such as inclusion bodies. They are usually described by the underlying cause.

$>$ Diabetic nephropathy: is a complication that occurs in some diabetics. Excess blood sugar accumulates in the kidney causing them to become inflamed and unable to carry out their normal function. This leads to the leakage of proteins into the urine.

$>$ Systemic lupus erythematosus: this autoimmune disease can affect a number of organs, among them the kidney, due to the deposit of immunocomplexes that are typical to this disease. The disease can also cause lupus nephritis.

$>$ Sarcoidosis: This disease does not usually affect the kidney but, on occasions, the accumulation of inflammatory granulomas (collection of immune cells) in the glomeruli can lead to nephrotic syndrome.

> Syphilis: kidney damage can occur during the secondary stage of this disease (between 2 and 8 weeks from onset).

$>$ Hepatitis B: certain antigens present during hepatitis can accumulate in the kidneys and damage them.

$>$ Sjogren's syndrome: this autoimmune disease causes the deposit of immunocomplexes in the glomeruli, causing them to become inflamed, this is the same mechanism as occurs in systemic lupus erythematosus.

$>$ HIV: the virus' antigens provoke an obstruction in the glomerular capillary's lumen that alters normal kidney function.

$>$ Amyloidosis: the deposit of amyloid substances (proteins with anomalous structures) in the glomeruli modifying their shape and function.

$>$ Multiple myeloma: the cancerous cells arrive at the kidney causing glomerulonephritis as a complication.

$>$ Vasculitis: inflammation of the blood vessels at a glomerular level impedes the normal blood flow and damages the kidney.

$>$ Cancer: as happens in myeloma, the invasion of the glomeruli by cancerous cells disturbs their normal functioning.

$>$ Genetic disorders: congenital nephrotic syndrome is a rare genetic disorder in which the protein nephrin, a component of the glomerular filtration barrier, is altered.

$>$ Drugs (like gold salts, penicillin, captopril): gold salts can cause a more or less important loss of proteins in urine as a consequence of metal accumulation. Penicillin is nephrotoxic in patients with kidney failure and captopril can aggravate proteinuria [6].

\section{By Histologic Pattern}

Membranous nephropathy (MN)

$>$ Sjogren's syndrome

$>$ Systemic lupus erythematosus (SLE)

$>$ Diabetes mellitus

$>$ Sarcoidosis

$>$ Drugs (such as corticosteroids, gold, intravenous heroin)

$>$ Malignancy (cancer) 
$>$ Bacterial infections, e.g. leprosy \& syphilis

$>$ Protozoal infections, e.g. malaria

Focal segmental glomerulosclerosis (FSGS)

$>$ Hypertensive nephrosclerosis

$>\mathrm{HIV}$

$>$ Obesity

$>$ Kidney loss

Minimal change disease (MCD)

$>$ Drugs, especially NSAIDs in the elderly

$>$ Malignancy, especially Hodgkin's lymphoma

$>$ Allergy

$>$ Bee sting

Membranoproliferative Glomerulonephritis

$>$ Hepatitis C

\section{Complications}

Nephrotic syndrome can be associated with a series of complications that can affect an individual's health and quality of life:

> Thromboembolic disorders: particularly those caused by a decrease in blood antithrombin III levels due to leakage. Antithrombin III counteracts the action of thrombin. Thrombosis usually occurs in the renal veins although it can also occur in arteries. Treatment is with oral anticoagulants (not heparin as heparin acts via anti-thrombin 3 which is lost in the proteinuria so it will be ineffective.) Hyper coagulopathy due to extravasation of fluid from the blood vessels (edema) is also a risk for venous thrombosis [7].

$>$ Infections: The increased susceptibility of patients to infections can be a result of the leakage of immunoglobulins from the blood, the loss of proteins in general and the presence of oedematous fluid (which acts as a breeding ground for infections). The most common infection is peritonitis, followed by lung, skin and urinary infections, meningoencephalitis and in the most serious cases septicaemia [8]. The most notable of the causative organisms are Streptococcus pneumoniae and Haemophilus influenzae.

$>$ Acute kidney failure due to hypovolemia: the loss of vascular fluid into the tissues (edema) produces a decreased blood supply to the kidneys that causes a loss of kidney function. Thus it is a tricky task to get rid of excess fluid in the body while maintaining circulatory euvolemia [9].

$>$ Pulmonary edema: the loss of proteins from blood plasma and the consequent fall in oncotic pressure causes an abnormal accumulation of liquid in the lungs causing hypoxia and dyspnoea [10].

$>$ Hypothyroidism: deficiency of the thyroglobulin transport protein thyroxin (a glycoprotein that is rich in iodine and is found in the thyroid gland) due to decreased thyroid binding globulin [11].

$>$ Hypocalcaemia: lack of 25-hydroxycholecalciferol (the way that vitamin D is stored in the body). As vitamin $\mathrm{D}$ regulates the amount of calcium present in the blood a decrease in its concentration leads to a decrease in blood calcium levels. It may be significant enough to cause tetany. Hypocalcaemia may be relative; calcium levels should be adjusted based on the albumin level and ionized calcium levels should be checked [12].

$>$ Microcytic hypochromic anaemia: iron deficiency caused by the loss of ferritin (compound used to store iron in the body). It is iron-therapy resistant.

$>$ Protein malnutrition: this occurs when the amount of protein that is lost in the urine is greater than that ingested, this leads to a negative nitrogen balance.

$>$ Growth retardation: can occur in cases of relapse or resistance to therapy. Causes of growth retardation are protein deficiency from the loss of protein in urine, anorexia (reduced protein intake), and steroid therapy (catabolism).

$>$ Vitamin D deficiency can occur. Vitamin D binding protein is lost $[13,14]$.

$>$ Cushing's Syndrome

\section{Epidemiology}

Nephrotic syndrome can affect any age, although it is mainly found in adults with a ratio of adults to children of $26: 1$.

The syndrome presents in different ways in the two groups: the most frequent glomerulopathy in children is minimal change disease ( $66 \%$ of cases), followed by focal and segmental glomeruloesclerosis (8\%) and mesangiocapillary glomerulonephritis (6\%). In adults the most common disease is mesangiocapillary glomerulonephritis (30-40\%), followed by focal and segmental glomeruloesclerosis (15-25\%) and minimal change disease (20\%). The latter usually presents as secondary and not primary as occurs in children. Its main cause is diabetic nephropathy. It usually presents in a patient's $40 \mathrm{~s}$ or $50 \mathrm{~s}$. Of the glomerulonephritis cases approximately $60 \%$ to $80 \%$ are primary, while the remainders are secondary $[15,16]$.

There are also differences in epidemiology between the sexes; the disease is more common in men than in women by a ratio of $2: 1$.

The epidemiological data also reveals information regarding the most common way that symptoms develop in patients with nephrotic syndrome: spontaneous remission occurs in up to $20 \%$ or $30 \%$ of cases during the first year of the illness. However, this improvement is not definitive as some $50 \%$ to $60 \%$ of 
patients die and / or develop chronic renal failure 6 to 14 years after this remission. On the other hand, between $10 \%$ and $20 \%$ of patients have continuous episodes of remissions and relapses without dying or jeopardizing their kidney. The main causes of death are cardiovascular, as a result of the chronicity of the syndrome, and thromboembolic accidents $[17,18]$.

\section{References}

1. Shamna, Jose J, Shijikumar, Ahmed R. A Brief Study of Nephrotoxicity and Nephroprotective Agents. Indian J. Pharm. Biol. Res. 2020; 8 (1): 9-13.

2. Kwan BC, Kronenberg F, Beddhu S, Cheung AK. Lipoprotein metabolism and lipid management in chronic kidney disease. J Am Soc Nephrol 2007; 18:1246.

3. Stubbs JR, Idiculla A, Slusser J, Menard R, Quarles LD. Cholecalciferol supplementation alters calcitriol-responsive monocyte proteins and decreases inflammatory cytokines in ESRD. J Am Soc Nephrol 2010;21:353-61.

4. Reinhard Brunkhorst. Nephrotic Syndrome. Urology at a Glance 2014:175-178.

5. Alchi B, Jayne D; Membranoproliferative glomerulonephritis. Pediatr Nephrol. 2010Aug;25(8):1409.

6. Hull RP,Goldsmith DJ. Nephrotic syndrome in adults. BMJ. 2008 May24;336(7654):1185-9.

7. Kodner $\mathrm{C}$;Nephrotic syndrome in adults: diagnosis and management.Am Fam Physician. 2009 Nov15;80(10):1129-34.

8. Basma H, Norrby-Teglund A, Low D, McGeer, El-Ahmady O, Kotb M. Risk factors in the pathogenesis of invasive group A streptococcal infections: role of protective humoral immunity. Infect Immun.1999;67:1871-1877.

9. Kakar Satinder, Kumar Vishal and Singh Ramandeep, Latest research progress on acute nephrotic syndrome, Journal of Acute Disease, 2017;6 (6):255-259.

\section{Source of Support: Nil}

Conflict of Interest: Nil
10. Bisno A L. Non-suppurative poststreptococcal sequelae: rheumatic fever and glomerulonephritis. In: Mandell G L, Bennett J E, Dolin R, editors. Principles and practice of infectious diseases.Vol. 2. New York, N.Y: Churchill Livingstone; 1995. pp. 1799-1810.

11. Sudha K , Anupama Hegde ,Poornima A Majrekar and Reshma Kumar Chandra. Prevalence of vitamin-D deficiency and its relationship with subclinical hypothyroidism. Int J Pharm Bio Sci 2013;4(4):(B)1380-84.

12. Usatii M, Rousseau L, Demers C, Petit JL, Brossard JH, Gascon-Barré M, et al. Parathyroid hormone fragments inhibit active hormone and hypocalcemia-induced $1.25(\mathrm{OH}) 2 \mathrm{D}$ synthesis. Kidney Int 2007;72:1330-5.

13. Xiang W, Kong J, Chen S, Cao LP, Qiao G, Zheng W, et al. Cardiac hypertrophy in vitamin D receptor knockout mice: role of the systemic and cardiac renin-angiotensin systems. Am J Physiol Endocrinol Metab 2005;288:E125-32.

14. Adams JS, Hewison M. Unexpected actions of vitamin D: new perspectives on the regulation of innate and adaptive immunity. Nat Clin Pract Endocrinol Metab 2008;4:80-90.

15. Hogan J, Radhakrishnan J. The treatment of minimal change disease in adults. JAm SocNephrol. 2013;24(5):702-11.

16. Mc Grogan A, Franssen CF, de Vries CS. The incidence of primary glomerulonephritis worldwide: a systematic review of the literature. Nephrol Dial Transplant. 2011;26(2):414-30.

17. J. W. Rich-Edwards, A. Fraser, D. A. Lawlor, and J. M. Catov, "Pregnancy characteristics and women's future cardiovascular health: an underused opportunity to improve women's health?" Epidemiologic Reviews, 2014;36 (1):57-70.

18. Mittal A, Sathian B, Kumar A, et al., The Clinical implications of thyroid hormones and its association with lipid profile: A comparative study from western Nepal, Nepal Journal of Epidemiology 2010;1(1):11-16. 